\title{
Systematic Comparison of Zwitterionic and Non-Zwitterionic Antifouling Polymer Brushes on a Bead-Based Platform
}

\author{
Esther van Andel, ${ }^{\dagger}{ }^{\ddagger}$ Stefanie C. Lange, ${ }^{\dagger}$ Sidharam P. Pujari, ${ }^{\dagger}$ Edwin J. Tijhaar, ${ }^{\ddagger}$

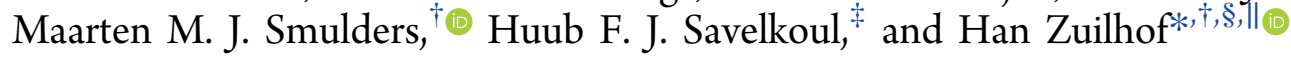 \\ ${ }^{\dagger}$ Laboratory of Organic Chemistry, Wageningen University, Stippeneng 4, 6708 WE Wageningen, The Netherlands \\ ${ }^{\ddagger}$ Cell Biology and Immunology Group, Wageningen University, De Elst 1, 6709 PG Wageningen, The Netherlands \\ ${ }^{\S}$ School of Pharmaceutical Sciences and Technology, Tianjin University, 92 Weijin Road, Tianjin 300072, People's Republic of \\ China \\ "Department of Chemical and Materials Engineering, King Abdulaziz University, Jeddah, Saudi Arabia
}

Supporting Information

\begin{abstract}
Nonspecific adsorption of biomolecules to solid surfaces, a process called biofouling, is a major concern in many biomedical applications. Great effort has been made in the development of antifouling polymer coatings that are capable of repelling the nonspecific adsorption of proteins, cells, and microorganisms. In this respect, we herein contribute to understanding the factors that determine which polymer brush results in the best antifouling coating. To this end, we compared five different monomers: two sulfobetaines, a carboxybetaine, a phosphocholine, and a hydroxyl acrylamide. The antifouling coatings were analyzed using our previously described bead-based method with flow cytometry as the read-out system. This method allows for the quick and automated analysis of thousands of beads per second, enabling fast analysis and good statistics. We report the first direct comparison made between a sulfobetaine with opposite charges separated by two and three methylene groups and a carboxybetaine bearing two separating methylene groups. It was concluded that both the distance between opposite charges and the nature of the anionic groups have a distinct effect on the antifouling performance. Phosphocholines and simple hydroxyl acrylamides are not often compared with the betaines. However, here we found that they perform equally well or even better, yielding the following overall antifouling ranking: HPMAA $\geq$ PCMA- $2 \approx$ CBMAA-2 > SBMAA-2 > SBMAA-3 $\gg$ nonmodified beads (HPMAA being the best).
\end{abstract}

\section{INTRODUCTION}

Nonspecific adsorption of biomolecules to surfaces is a major concern in many applications, including drug-delivery systems, medical implants, and diagnostic devices. ${ }^{1,2}$ Compromised sensitivity of diagnostic tests ${ }^{3}$ and adverse immune responses against drug-delivery carriers and indwelling medical devices ${ }^{4}$ illustrate the great need for effective nonfouling materials.

Two decades ago, Whitesides and co-workers performed a systematic study on the efficiency of different monolayers to suppress protein adsorption, ${ }^{5,6}$ leading to a set of empirical guidelines that is now often referred to as the "Whitesides rules". ${ }^{7}$ These guidelines state that good antifouling layers have (1) polar functional groups, i.e., are hydrophilic, (2) hydrogen bond acceptors, (3) no hydrogen bond donors, and (4) zero net charge. Many different types of antifouling materials have been developed that follow these guidelines of which poly(ethylene glycol) (PEG) based layers are probably the most widely studied and used. ${ }^{8,9}$ Despite their frequent use and ability to prevent protein adsorption from single protein solutions, their antifouling capability is limited for use with complex biological media such as blood plasma and blood serum. ${ }^{10}$ As an attractive alternative to PEG, zwitterionic materials have been extensively studied due to their stability in aqueous solutions, ${ }^{11}$ biocompatibility, ${ }^{12}$ and excellent antifouling properties even in complex biological media. ${ }^{10,13}$ Carboxybetaine (CB) and sulfobetaine (SB) monomers are most commonly used to graft zwitterionic brushes from surfaces because of their commercial availability and straightforward synthesis. ${ }^{14,15}$ Next to that, phosphocholinebased (PC) polymers are the only zwitterionic antifouling materials that are FDA approved and used to enhance the performance of medical devices ${ }^{16}$ and thereby form another important class of antifouling materials. Interestingly, nonzwitterionic materials like simple hydroxyl-containing mono-

Special Issue: Zwitterionic Interfaces: Concepts and Emerging Applications

Received: May 31, 2018

Revised: August 30, 2018

Published: September 28, 2018 
mers have also been shown to perform really well even in complex media, ${ }^{17-19}$ even though these materials do not follow the "Whitesides rules" by being only moderately hydrophilic and containing hydrogen bond donors.

In the past decade, initial systematic studies have attempted to reveal the exact relationship between monomer structure and antifouling performance. ${ }^{10,14,17,20-25}$ These studies revealed that even small changes in monomer structure can influence the antifouling performance of the resulting polymer brushes quite significantly. Important factors include, but are not limited to, type of polymerizable group (methacrylate, acrylate, methacrylamide), ${ }^{17}$ nature of the hydrophilic groups (hydroxyl, quaternary ammonium, sulfonate, carboxylate, phosphonate), ${ }^{10,17,26,27}$ and the carbon spacer length (CSL), ${ }^{14,17,22,24,25}$ which is defined as the number of methylene groups between the cationic and anionic groups (or between hydroxyl and acrylamide). Despite such studies, a general understanding of the ultimate antifouling material has not yet been reached. Most systematic studies focused either on carboxybetaines or sulfobetaines (or a combination thereof) or on hydroxyl-containing polyacrylamides. Direct comparisons of any betaines with the hydroxyl monomers is scarce. In addition, PC-based materials are rarely the topic of systematic studies, probably because of their challenging synthesis. ${ }^{16,28}$ Studies including sulfobetaines typically only consider SB-3 (a sulfobetaine with three methylene groups between opposite charges). Although SB-3 shows good protein resistance, its antifouling efficiency is not as high as observed for CB-2. ${ }^{10}$ This can be explained by their different CSLs; moreover, differences in hydration between the anionic groups in CB and SB monomers have also been reported. ${ }^{26}$ Two recent studies compared the effect of the CSL in sulfobetaines on their hydration states and fouling behavior for different ionic strengths and types of ions. ${ }^{24,25}$ It was concluded that under nonphysiological, low ionic strength conditions (i.e., $10^{-3}$ to $10^{-1} \mathrm{M}$, whereas isotonic phosphate-buffered saline (PBS) and blood serum are typically $>0.15 \mathrm{M})$, poly (SB-2) surfaces performed less well regarding prevention of nonspecific protein adsorption than poly(SB-3). This was attributed to the stronger intra/interchain interactions within the poly(SB-2) brushes, resulting in less hydrated polymer layers. ${ }^{25}$ The fact that SB-2 monomers were, to our knowledge, never related to SB-3 and CB-2 monomers under the physiological conditions for which zwitterionic materials have shown to be so useful, prompted us to directly compare polymer brushes derived from the SB-3, SB-2, and CB-2 monomers and thereby deepen our understanding of the structural dependence of monomers on the antifouling performances of the resulting polymer brushes. To make a more comprehensive comparison to other main classes of well-performing polymers, polymer brushes derived from a PC and a hydroxyl acrylamide monomer were also included in the study.

Herein, we compare the antifouling performance of polymer brushes derived from two sulfobetaines, SBMAA-3 and SBMAA-2, the carboxybetaine CBMAA-2, the phosphocholine PCMA-2 and, the hydroxyl acrylamide HPMAA (Scheme 1). Polymer brushes were grown and evaluated using our previously described bead-based platform. In this method, beads are coated with a polymer brush using standard surfaceinitiated atom transfer radical polymerization (SI-ATRP) conditions, and the adsorption of proteins on these coated brushes from a purified protein (bovine serum albumin (BSA)) solution and from a complex protein solution (bovine serum)
Scheme 1. Schematic Overview of Stepwise Polymer Brush Formation from Gold Surfaces and Dynabeads ${ }^{a}$
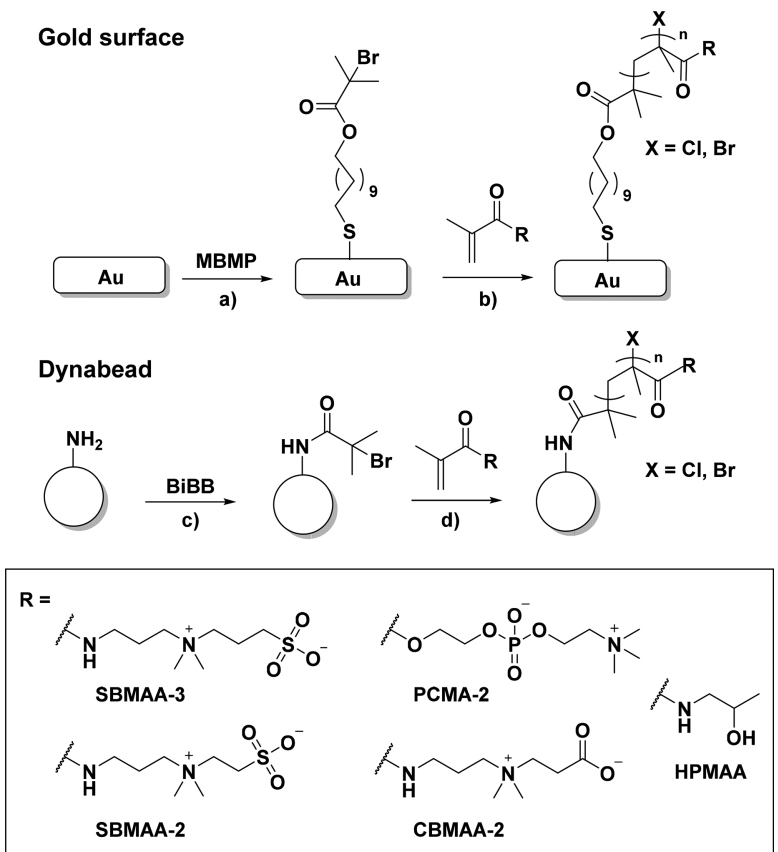

${ }^{a}$ Notes: (a) MBMP, EtOH, $24 \mathrm{~h}, \mathrm{rt}$; (b and d) for SBMAA-3, SBMAA-2, CBMAA-2, and PCMA-2: $\mathrm{Cu}(\mathrm{I}) / \mathrm{Cu}(\mathrm{II})$ (90/10), 2,2' bipyridyl, isopropanol/water (20/80), $12 \mathrm{~min}$, rt, for HPMAA: $\mathrm{Cu}(\mathrm{I}) / \mathrm{Cu}(\mathrm{II})(90 / 10), \mathrm{Me}_{4} \mathrm{Cyclam}, \mathrm{EtOH}, 2.5 \mathrm{~h}, 30{ }^{\circ} \mathrm{C}$; (c) $\alpha$ bromoisobutyryl bromide, DIPEA, DCM, overnight, rt.

is measured using flow cytometry. ${ }^{29}$ As this technique measures thousands of beads, rather than individually modified surfaces, this allows statistical rigor, exactly as is required for the systematic assessment of the different monomers. The study yielded a clear order of the antifouling capacity of the derived brushes and detailed insights into the influence of the various factors involved in different media.

\section{MATERIALS AND METHODS}

Materials. All chemicals and solvents were used without further purification. Acetone (HPLC grade; 99.9\%, BIOSOLVE BV), $N, N$ dimethylformamide (DMF) (Fisher Scientific), methanol $(\mathrm{MeOH})$ (HPLC grade, Rathburn Chemicals Ltd.), dichloromethane (DCM) (VWR International S.A.S.), isopropanol (HPLC grade; BIOSOLVE), absolute ethanol (EtOH) (Fisher Scientific), dry tetrahydrofuran (Sigma-Aldrich), and deionized water produced with a Milli-Q Integral 3 system (Millipore, Molsheim, France) were used as solvents. $\mathrm{N}$-(3-(Dimethylamino)propyl)methacrylamide (DMAPMAA) (99\%), sodium 2-bromoethane-1-sulfonate (98\%), N,Ndiisopropylethylamine (DIPEA), copper(I)chloride ( $\geq 99 \%)$, copper(II)chloride (97\%), copper(I)bromide (99.999 trace metals basis) copper(II)bromide (99.999\% trace metals basis), 2,2'-bipyridyl (99\%), $\alpha$-bromoisobutyryl bromide (98\%), $\beta$-propiolactone, 1,1 diphenyl-2-picryl hydrazyl (DPPH) (97\%), 1,4,8,11-tetramethyl1,4,8,11-tetraazacyclotetradecane $\left(\mathrm{Me}_{4} \mathrm{Cyclam}\right)$ (98\%), and 2-methacryloyloxyethyl phosphocholine (PCMA-2) (97\%) were all purchased from Sigma-Aldrich. 1,3-Propane sultone was ordered from Alfa Aesar (99\%), and 11-mercaptoundec-1-yl 2-bromo-2-methylpropionate (MBMP) was purchased from Prochimia. Bovine serum albumin-Alexa Fluor 488 conjugate (BSA-AF488) and EZ-Link SulfoNHS-LC-Biotin were obtained from Thermo Fisher and streptavidinphycoerythrin (PE) conjugate from eBioscience. AnaTag HiLyte Fluor 488 microscale protein labeling kit was obtained from AnaSpec, Inc. PD10 desalting columns were bought from GE Healtcare; flat 
gold substrates of $\mathrm{Au}$ sputtered on glass $(1 \times 1 \mathrm{~cm})$ were purchased from Xantec and magnetic Dynabeads (Dynabeads M-270 amine, 2.8 $\mu \mathrm{m}$ in diameter) were purchased from Invitrogen Life Technologies. The $N$-(2-hydroxypropyl)methacrylamide (HPMAA) monomer was obtained from Polysciences, Inc. Phosphate-buffered saline (PBS) buffer $\mathrm{pH} 7.4\left(5.4 \mathrm{mM} \mathrm{Na}_{2} \mathrm{HPO}_{4} \cdot 2 \mathrm{H}_{2} \mathrm{O}, 1.3 \mathrm{mM} \mathrm{KH}_{2} \mathrm{PO}_{4}, 154 \mathrm{mM}\right.$ $\mathrm{NaCl}$ ) was used for all experiments, unless otherwise stated.

Synthesis of SBMAA-2, SBMAA-3, and CBMAA-2. SBMAA-3. 3-((3-Methacrylamidopropyl)dimethylammonio)propane-1-sulfonate (SBMAA-3) was synthesized based on previously reported methods. ${ }^{30}$ First, $12.2 \mathrm{~g}(100 \mathrm{mmol})$ of 1,3-propane sultone was dissolved in 150 $\mathrm{mL}$ of acetone and cooled to $0{ }^{\circ} \mathrm{C}$. After adding $10.0 \mathrm{~mL}$ of $(55.0$ mmol) DMAPMAA, the reaction mixture was allowed to warm to room temperature and stirred overnight. The resulting precipitate was filtered off and washed rigorously with $500 \mathrm{~mL}$ of acetone. After drying under high vacuum, the desired product was obtained as a white powder ( $15.1 \mathrm{~g}, 51.7 \mathrm{mmol}, 94 \%)$. The monomer was stored in the fridge at $4{ }^{\circ} \mathrm{C}$. (For detailed characterization, see the Supporting Information.)

SBMAA-2. For the synthesis of 2-((3-methacrylamidopropyl)dimethylammonio)ethane-1-sulfonate (SBMAA-2), $5.0 \mathrm{~g}$ of DMAPMAA $(29 \mathrm{mmol})$ and $6.2 \mathrm{~g}$ of 2-bromoethanesulfonate $(29 \mathrm{mmol})$ were added to a $250 \mathrm{~mL}$ round-bottom flask. The reagents were dissolved in $100 \mathrm{~mL}$ of DMF under a nitrogen atmosphere and vigorous stirred at $60{ }^{\circ} \mathrm{C}$ overnight. Next, $5.1 \mathrm{~mL}$ of DIPEA (43 mmol) was added, and $2 \mathrm{~h}$ later, another equivalent of 2 bromoethanesulfonate was added. The reaction mixture was stirred for an additional 3 days at $70{ }^{\circ} \mathrm{C}$. DMF was removed under reduced pressure, and the resulting mixture was filtered over a plug of silica using EtOH:DCM (1:1) followed by flushing with $\mathrm{MeOH}$ to elute the product. A second column using $100 \% \mathrm{MeOH}$ yielded the product as a white solid ( $4.5 \mathrm{~g}, 16 \mathrm{mmol}, 56 \%$ isolated yield). The product was stored in the freezer at $-20{ }^{\circ} \mathrm{C}$. (For detailed characterization, see the Supporting Information.)

CBMAA-2. The synthesis of 3-((3-methacrylamidopropyl)dimethylammonio)propanoate (CBMAA-2) was based on the procedure of Rodriguez-Emmenegger et al. ${ }^{31}$ First, $47.2 \mathrm{~g}$ (277 mmol) of DMAPMAA and a small amount diphenylpicryl hydrazyl (DPPH) as inhibitor were dissolved in $250 \mathrm{~mL}$ of dry THF and cooled to $0{ }^{\circ} \mathrm{C}$. Then, $25.0 \mathrm{~g}(347 \mathrm{mmol})$ of $\beta$-propiolactone was dissolved in $50 \mathrm{~mL}$ of dry THF and dropwise added to the DMAPMAA solution. After $3 \mathrm{~h}$ at $0{ }^{\circ} \mathrm{C}$, stirring was continued for 24 $\mathrm{h}$ at $4{ }^{\circ} \mathrm{C}$. The formed precipitate was filtered off over a glass filter, and the filtrate was washed with THF and ether. The product was dissolved in $\mathrm{MeOH}$ and subsequently precipitated in THF to yield a white solid ( $30.0 \mathrm{~g}, 124 \mathrm{mmol}, 45 \%$ yield). The product was stored in the freezer at $-20{ }^{\circ} \mathrm{C}$. (For detailed characterization, see the Supporting Information.)

Initiator Attachment. Au Surfaces. Prior to use, Au surfaces (Xantec) were rinsed with $\mathrm{EtOH}$, Milli-Q water, and $\mathrm{EtOH}$ again followed by drying in a stream of argon. The Au surfaces were immersed in a $2.5 \mu \mathrm{L} / \mathrm{mL}$ solution of 11-mercaptoundec-1-yl 2bromo-2-methylpropionate (MBMP) in absolute EtOH. The immersed surfaces were placed on a shaker $(80 \mathrm{rpm})$ at room temperature for $24 \mathrm{~h}^{32}$ The initiator-modified surfaces were cleaned by rinsing with, and sonicating in, $\mathrm{EtOH}$ followed by rinsing with EtOH, Milli-Q water, EtOH, and DCM and drying in a stream of argon.

Beads. Amine-terminated Dynabeads (M-270 Amine) were functionalized with an ATRP initiator based on a previously described procedure. $^{29}$ In short, $600 \mu \mathrm{L}$ of Dynabeads were dried in a vacuum oven at $50{ }^{\circ} \mathrm{C}$ for at least $2 \mathrm{~h}$. The beads were resuspended in $2 \mathrm{~mL}$ of dry DCM and $0.5 \mathrm{~mL}(2.9 \mathrm{mmol})$ of DIPEA before $0.6 \mathrm{~mL}(4.9$ mmol) of $\alpha$-bromoisobutyryl bromide was added. The reaction flask was wrapped with aluminum foil and placed overnight on an endover-end shaker at room temperature. The beads were washed with a copious amount of DCM, then washed twice with $1 \mathrm{~mL}$ of isopropanol and twice with $1 \mathrm{~mL}$ of Milli- $\mathrm{Q}$ water. The beads were used the same day for surface-initiated polymerization.
Surface-Initiated Polymerization of SBMAA-3, SBMAA-2, CBMAA-2, and PCMA-2. Surface-initiated atom transfer radical polymerization (SI-ATRP) was performed according to previously described procedures ${ }^{29,33}$ with slight modifications. All steps were performed under an argon atmosphere in Schlenk flasks, and solutions were transferred using argon-flushed syringes.

Au Surfaces. An isopropanol/Milli-Q water mixture (20/80) was degassed by $5 \mathrm{~min}$ of sonication and $30 \mathrm{~min}$ of argon bubbling. Then, $16.1 \mathrm{mg}(0.16 \mathrm{mmol})$ of a $\mathrm{Cu}(\mathrm{I}) \mathrm{Cl} / \mathrm{Cu}(\mathrm{II}) \mathrm{Cl}_{2}(90 / 10)$ mixture and $54.7 \mathrm{mg}(0.35 \mathrm{mmol})$ of 2,2'-bipyridyl were dissolved in $7.0 \mathrm{~mL}$ of the isopropanol/Milli- $Q$ mixture and stirred for $15 \mathrm{~min}$. From the resulting brown solution, $1.1 \mathrm{~mL}$ was transferred per flask containing $1.00 \mathrm{mmol}$ of the appropriate monomer (292 mg of SBMAA-3, 278 mg of SBMAA-2, $242 \mathrm{mg}$ of CBMAA-2, and $295 \mathrm{mg}$ of PCMA-2, respectively). After $15 \mathrm{~min}$ of stirring, except for PCMA for which sample handling was performed as quick as possible to avoid polymerization in solution, the reaction mixtures were transferred to the initiator-modified gold surfaces. The surfaces were placed separately and diagonally in Schlenk flasks with the modified surfaces facing downward, enabling stirring underneath the surfaces and avoiding sedimentation of solids on the reactive side of the chip. The polymerization reactions were carried out for $12 \mathrm{~min}$ at room temperature while the flasks were covered with aluminum foil. The reactions were stopped by rinsing with, and sonicating in, $60^{\circ} \mathrm{C}$ Milli$\mathrm{Q}$ water for $5 \mathrm{~min}$. The surfaces were rinsed with acetone and dried in a stream of argon. Surfaces were stored in an argon glovebox until further use.

Beads. SI-ATRP on beads was performed similarly as on the abovedescribed gold surfaces with the following exceptions: $23.0 \mathrm{mg}(0.23$ $\mathrm{mmol})$ of the $\mathrm{Cu}(\mathrm{I}) \mathrm{Cl} / \mathrm{Cu}(\mathrm{II}) \mathrm{Cl}_{2}(90 / 10)$ mixture and $78.1 \mathrm{mg}(0.5$ $\mathrm{mmol}$ ) of $2,2^{\prime}$-bipyridyl were dissolved in $8.2 \mathrm{~mL}$ of degassed isopropanol/Milli-Q. To each monomer was added $900 \mu \mathrm{L}$ of the $\mathrm{Cu}-$ Bpy mixture, which was subsequently transferred to a flask containing initiator-modified beads (amount of beads comparable to $100 \mu \mathrm{L}$ of nonmodified beads) dispersed in $200 \mu \mathrm{L}$ of the isopropanol/Milli-Q mixture. The reaction was carried out in an aluminum-covered Schlenk flask, which was placed under a $45^{\circ}$ angle on a shaker $(80$ $\mathrm{rpm})$. The reaction was stopped by collecting the beads with a magnetic stand (Promega) and washing them with copious amounts of Milli-Q water, twice with phosphate-buffered saline (PBS pH 7.4), and then again twice with Milli- $Q$ water. The beads were stored in Milli- $Q$ water in a refrigerator $\left(4^{\circ} \mathrm{C}\right)$ until further use.

Surface-Initiated Polymerization of HPMAA. SI-ATRP using HPMAA was adapted from a protocol developed by RodriguezEmmenegger et al. ${ }^{19}$ All steps were performed under an argon atmosphere and using Schlenk flasks. Absolute EtOH and initiatorfunctionalized Dynabeads in $0.5 \mathrm{~mL}$ of $\mathrm{EtOH}$ were degassed by performing eight freeze-pump-thaw cycles using liquid nitrogen to freeze and $30{ }^{\circ} \mathrm{C}$ water to thaw. To a mixture of $3.1 \mathrm{mg}(14 \mu \mathrm{mol})$ of $\mathrm{CuBr}_{2}, 9.6 \mathrm{mg}(67 \mu \mathrm{mol})$ of $\mathrm{CuBr}$, and $20.5 \mathrm{mg}(80 \mu \mathrm{mol})$ of $\mathrm{Me}_{4} \mathrm{Cyclam}$ was added $4 \mathrm{~mL}$ of degassed $\mathrm{EtOH}$. After rigorous stirring and a short sonication step, the resulting bright blue reaction mixture was transferred to a flask containing $480 \mathrm{mg}(3.4 \mathrm{mmol})$ of HPMAA, and this mixture was stirred until HPMAA was fully dissolved. To the beads and to an initiator-modified gold surface in $0.5 \mathrm{~mL}$ of degassed EtOH was added $2 \mathrm{~mL}$ of HPMAA solution to a final volume of $2.5 \mathrm{~mL}$. The flasks were placed on a shaker (at a $45^{\circ}$ angle, shaking at $80 \mathrm{rpm}$ ) covered in aluminum foil and allowed to react for $2.5 \mathrm{~h}$ at $30{ }^{\circ} \mathrm{C}$. The surface and beads were washed with $\mathrm{EtOH}$, and the surface was also sonicated in $\mathrm{EtOH}$ for 5 min and rinsed with Milli-Q water.

Surface Characterization. Static Water Contact Angle (SCA). The wettability of modified gold surfaces was determined by automated static water contact angle measurements using a Krüss DSA-100 goniometer. Droplets of Milli-Q water $(3 \mu \mathrm{L})$ were dispensed on the surface, and angles were measured with a CCD camera using a tangential method.

$X$-ray Photoelectron Spectroscopy (XPS). Prior to XPS analysis, modified beads (in Milli-Q) were dropcast on a plasma-cleaned piece of $\mathrm{Si}(111)$ and dried in a vacuum oven at $50{ }^{\circ} \mathrm{C}$ for at least $2 \mathrm{~h} .{ }^{29}$ 
Dropcast beads and modified gold surfaces were subsequently analyzed using a JPS-9200 photoelectron spectrometer (JEOL, Japan). The spectra were obtained using monochromatic $\mathrm{Al} \mathrm{K \alpha} \mathrm{X}$ ray radiation at $12 \mathrm{kV}$ and $20 \mathrm{~mA}$ with an analyzer energy pass of 10 $\mathrm{eV}$ for narrow scans. The obtained spectra were processed using the CASA XPS peak fit program (version 2.3.16 PR 1.6).

Polymer brush layer thicknesses on gold surfaces were calculated using the equation ${ }^{32,34} d=\ln \left(I_{\mathrm{Au} 0}\right) / I_{\mathrm{Au}} \cdot \lambda_{\mathrm{Au}} \cdot \cos \theta$, where $d=$ thickness (in $\mathrm{nm}$ ) of the organic layer, $I_{\mathrm{Au} 0}=$ intensity of the XPS signal of $\mathrm{Au}$ $4 \mathrm{f}_{7 / 2}$ at $83.9 \mathrm{eV}$ (relative to $\mathrm{C} 1 \mathrm{~s}$ signal) in unmodified gold, $I_{\mathrm{Au}}=$ intensity of XPS signal of $A u 4 f_{7 / 2}$ (relative to $C 1$ s signal) in modified gold, $\lambda_{\mathrm{Au}}=$ effective attenuation length of $\mathrm{Au} 4 \mathrm{f}$ electrons in the organic films (using a value of $3.858 \mathrm{~nm}$ as reported by Petrovykh et $\left.\mathrm{al}^{35}\right)$, and $\theta=$ the photoelectron emission takeoff angle relative to the surface normal $\left(10^{\circ}\right)$.

Zeta Potential. Zeta $(\zeta)$ potential measurements were performed using a Zetasizer Nano-ZS apparatus (Malvern Panalytical) equipped with a $\mathrm{He} \mathrm{Ne}$ laser operating at $633 \mathrm{~nm}$ and a dip cell with palladium electrodes (ZEN1002, Malvern Panalytical). Bead suspensions were prepared in PBS pH 7.4 ( $1 \mathrm{~mL}$ total), vortexed prior to use, and transferred to disposable cuvettes (PS $2.5 \mathrm{~mL}$, CAT No. 7590, BRAND). Measurements were performed at $25{ }^{\circ} \mathrm{C}$. The Zetasizer Malvern ver. 7.02 software was used to acquire the data. The voltage was manually set to $4 \mathrm{~V}$ and used in combination with monomodal measurement settings (to allow measurements in PBS). Measurements were performed using 2 min equilibration time, $10-100$ runs (automatically determined) per measurement, five subsequent measurements per sample and using Smoluchowski's model to determine the zeta potentials. All measurements were carried out in duplicate.

Serum Labeling. Bovine serum was obtained and biotinylated as previously described. ${ }^{29}$ Bovine blood serum samples were obtained from healthy cows via coccygeal vein sampling. Serum was collected using Vacuette Blood Collection tubes $(4 \mathrm{~mL}$ of $\mathrm{Z}$ Serum Separator Clot Activator $13 \times 75$ gold cap-gold ring, premium) from Greiner Bio-One. Blood sample collection was approved by the Board on Animal Ethics and Experiments from Wageningen University (DEC number: 2014005.b). Serum samples (from three different cows) were combined and heated for $30 \mathrm{~min}$ at $56{ }^{\circ} \mathrm{C}$ in a water bath to inactivate any immunologically active complement proteins. Serum samples were stored at $-20{ }^{\circ} \mathrm{C}$ and prior to use were thawed and centrifuged at $9000 \mathrm{~g}$ for $2 \mathrm{~min}$.

Serum proteins were biotinylated using an EZ-Link Sulfo-NHS-LCBiotin reagent using the manufacturer's instructions. Assuming that the average molecular weight of serum proteins is $70 \mathrm{kDa}, 50$ equiv of sulfo-NHS-biotin to serum proteins was used. The reaction was carried out at room temperature for $60 \mathrm{~min}$. Nonreacted material was removed using a desalting PD-10 column (GE Healthcare), following the manufacturer's gravitation protocol with PBS as eluent. The concentration of the obtained biotinylated serum (Serum-biotin) was adjusted with PBS to $10 \%$ serum solution (i.e., $\sim 6 \mathrm{mg}$ of total protein $/ \mathrm{mL}$ ).

Antifouling Studies. Flow Cytometry. For each type of polymercoated beads, three samples were prepared: beads incubated in PBS, beads incubated in a BSA-AF488 $(0.5 \mathrm{mg} / \mathrm{mL}$ in PBS) solution, and beads incubated in $10 \%$ biotinylated serum. To a $2 \mathrm{~mL}$ Eppendorf tube were added and mixed $200 \mu \mathrm{L}$ of the appropriate solution and 2 $\mu \mathrm{L}$ of the appropriate bead dispersion. The tubes were covered with aluminum foil and placed on an end-over-end shaker for $30 \mathrm{~min}$ at room temperature. The beads were collected using a magnetic stand and washed three times with PBS. The beads that were incubated with biotinylated serum were subsequently incubated for $30 \mathrm{~min}$ with a streptavidin-phycoerythrin conjugate (Strep-PE, 1:50 dilution) followed by washing three times with PBS. After the last washing step, the beads were resuspended in $0.5 \mathrm{~mL}$ of PBS and transferred to FACS tubes. The beads were analyzed with a BD FACS Canto A (BD Biosciences) flow cytometer. Per sample, 10,000 single beads were measured. BSA-AF488 was visualized in the FITC-channel and Strep$\mathrm{PE}$ in the PE channel. Data analysis was performed using FlowJo software V10.
Surface Plasmon Resonance. Poly(SBMAA-2)- and poly(SBMAA-3)-functionalized gold surfaces were glued onto sample holders (Xantec) using super glue gel (Bison). Protein adsorption was measured by SPR using a Biacore 3000 (GE Healthcare) at $25{ }^{\circ} \mathrm{C}$ with a constant flow of $2 \mu \mathrm{L} / \mathrm{min}$. Protein adsorption was monitored by first wetting the surfaces by flowing runner buffer (PBS) for $30 \mathrm{~min}$ followed by injecting a BSA-containing solution $(1 \mathrm{mg} / \mathrm{mL})$ or dialyzed bovine blood serum (10, 33, and $100 \%$ in PBS) for $20 \mathrm{~min}$ followed by washing with running buffer. The response obtained after injection relative to the starting baseline was taken as a measure for the amount of protein fouling. To correct for baseline drifts, linear background subtraction was applied (Origin version 8.5) to all obtained sensorgrams.

\section{RESULTS AND DISCUSSION}

Synthesis of SBMAA-3, SBMAA-2, and CBMAA-2. Phosphocholine (PCMA-2) and hydroxyl methacrylate (HPMAA) are commercially available, while the sulfobetaines (SBMAA-3 and SBMAA-2) and carboxybetaine (CBMAA-2) monomers had to be synthesized. Zwitterionic betaines are typically prepared via a ring-opening reaction of a sultone or a lactone with an acrylate or acrylamide like $\mathrm{N}$-(3(dimethylamino)propyl) acrylamide (DMAPMAA) to yield the desired sulfobetaine or carboxybetaine, respectively. ${ }^{36}$ The carbon spacer length between the opposite charges of the resulting betaines can be tuned by changing the size of the ring. Various one-step ring-opening syntheses have been described with high yield and easy purification. ${ }^{30,31,37}$ SBMAA-3 and CBMAA-2 were synthesized in a similar fashion using DMAPMAA with 1,3-propane sultone ${ }^{30}$ and 1,3-propiolactone, ${ }^{31}$ respectively. Following this synthetic approach, a sultone with two methylene groups, a $\beta$-sultone, should yield the SBMAA- 2 monomer. However, it has been reported that $\beta$ sultones are too unstable to be isolated. ${ }^{38}$ Two alternative approaches have been described to obtain a sulfobetaine with a carbon spacer length of two: the Michael addition of ethenesulfinyl chloride with $N, N$-dimethylaminoethyl methacrylate $^{36}$ and the nucleophilic substitution reaction of 2bromoethanesulfonic acid with DMAPMAA. ${ }^{25}$ We improved the latter approach with respect to yield and purity by adding reactant and base in a stepwise manner and by performing two columns to subsequently isolate and purify the product. The thus acquired SBMAA-2 monomer was obtained in an improved yield of $56 \%$ at a $5 \mathrm{~g}$ scale. For full characterization (including ${ }^{1} \mathrm{H},{ }^{13} \mathrm{C}$, GHSQC, and GCOSY NMR and IR and ESI), see the Supporting Information (Figures S1-S4).

Modification of Gold Surfaces. For establishing the right polymerization conditions to obtain brushes of equal thickness for each of the monomers, polymer brushes were first grown from gold-coated glass chips, after which the reaction conditions were transferred to functionalize microbeads (Scheme 1). Polymer brush-coated gold substrates allowed the determination of wetting properties and layer thicknesses of the grafted polymer brushes. For this purpose, the surfaces were functionalized with an ATRP initiator via the selfassembly of 11-mercaptoundec-1-yl 2-bromo-2-methylpropionate (MBMP) on the gold layer. SI-ATRP was subsequently performed to grow polymer brushes from the surface using SBMAA-3, SBMAA-2, CBMAA-2, PCMA-2, or HPMAA as monomer. The zwitterionic brushes were grown following our previously reported procedure, ${ }^{33}$ while the poly(HPMAA) brushes were grown as described by Rodriguez-Emmenegger and co-workers. ${ }^{19}$ The XPS wide scan spectra (see also Figures S13A, S15A, S17A, and S19A for quantitative analysis), water 

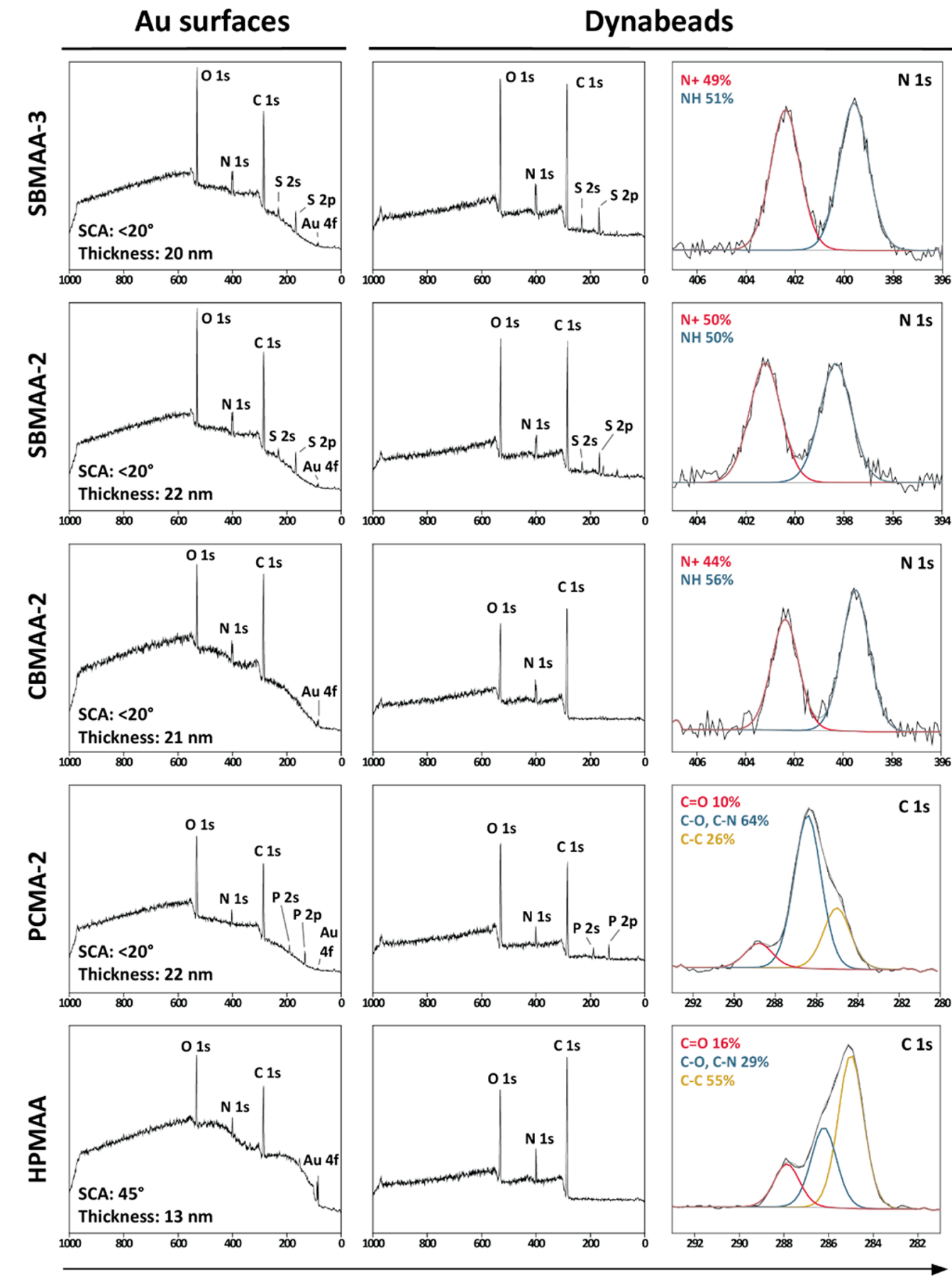

Binding energy (eV)

Figure 1. Overview of gold $\mathrm{Au}$ ) surfaces and Dynabeads modified with SBMAA-3, SBMAA-2, CBMAA-2, PCMA-2, or HPMAA polymer brushes, respectively. Reported thicknesses are based on the average of two measurements and based on XPS Au/C ratios. Wide scan spectra and $\mathrm{N} 1 \mathrm{~s}$ and C 1s spectra are depicted for the modified beads.

contact angles and polymer thicknesses of the obtained surfaces are shown in Figure 1, whereas the XPS narrow scan spectra are presented in the Supporting Information (Figures S13, S15, S17, and S19).

The XPS wide scan spectra show the expected elements for each type of polymer brush. That is, besides oxygen, nitrogen, and carbon for the poly(HPMAA) and poly(CBMAA-2) layers, also phosphorus (133 eV for P 2p, $190 \mathrm{eV}$ for P 2s) for the phosphocholine-based brushes and sulfur ( $166 \mathrm{eV}$ for S $2 \mathrm{p}$, $230 \mathrm{eV}$ for $\mathrm{S} \mathrm{2s}$ ) for the sulfobetaine-based brushes were detected at the expected ratios. Moreover, the carboxy- (Figure S17C) and sulfobetaines (Figure S13C, S15C) show the characteristic 1:1 ratio of the ammonium (401-402 eV) and amide nitrogen $(398-399 \mathrm{eV})$ in the $\mathrm{N}$ 1s narrow scan spectra, confirming the successful growth of intact betaine-based coatings. The poly(HPMAA) and poly(PCMA-2) brushes show only one peak in the N 1s narrow scans (Figure S19 and S21), which corresponds to either the quaternary ammonium (PCMA-2, $401 \mathrm{eV}$ ) or the amide nitrogen (HPMAA, $400 \mathrm{eV}$ ). For all five tested monomers, a distinct carbonyl, $\mathrm{C}-\mathrm{N} / \mathrm{C}-\mathrm{O}$, 
and $\mathrm{C}-\mathrm{C}$ peak could be observed in the XPS C 1s spectra at 288-289, 286 and $285 \mathrm{eV}$, respectively.

In each wide scan spectrum, a gold ( $\mathrm{Au} 4 \mathrm{f}$ ) peak is visible at $85 \mathrm{eV}$, allowing us to calculate the thicknesses of the coatings. The zwitterionic polymer brushes were grown under identical conditions and reaction times ( $\sim 12 \mathrm{~min})$, which lead in all cases to brush thicknesses between 20 and $22 \mathrm{~nm}$. Polymer thicknesses of $\sim 20 \mathrm{~nm}$ have been shown to be proficient to obtain excellent antifouling performances. ${ }^{39}$ The polymerization of HPMAA turned out to be nonoptimal using our standard polymerization method in water. Therefore, a modified procedure using $\mathrm{Me}_{4}$ Cyclam as ligand and $\mathrm{EtOH}$ as solvent was used. ${ }^{19}$ Using the $\mathrm{Me}_{4}$ Cyclam/EtOH protocol, a layer of $13 \mathrm{~nm}$ was obtained for the poly(HPMAA) brushes. It has been known that ATRP reactions carried out in less polar solvents $(\mathrm{EtOH})$ are slower than ATRP reactions performed in water, ${ }^{40}$ explaining why after $2.5 \mathrm{~h}$ at $30{ }^{\circ} \mathrm{C}$ thinner brushes were obtained for the HPMAA monomer.

The static water contact angle of the zwitterionic layers were all found to be below $20^{\circ}$, showing their excellent wetting behavior that is often described as key for good antifouling performance. $^{2}$ A water contact angle of $45^{\circ}$ was found for the poly(HPMAA) layers, which is in good agreement with previously reported HPMAA-based coatings, ${ }^{19,41}$ suggesting a sufficiently thick brush for good antifouling properties. The contact angle is in line with the hydrophilic nature of the monomer but of course not as low as those obtained for the zwitterionic coatings.

Modification of Microbeads. Magnetic Dynabeads were coated with poly(SBMAA-3), poly(SBMAA-2), poly(CBMAA2), poly(PCMA-2), or poly(HPMAA) brushes via an SI-ATRP procedure that was adapted to beads ${ }^{29}$ but similar in terms of concentrations and reaction times compared to the brushes grown on the flat gold surfaces. As the ATRP procedures used on gold surfaces resulted in polymer brushes of similar thicknesses for all monomers, it can be assumed that the brushes on the beads will also result in comparable thicknesses for the different monomers. To modify the beads, an ATRP initiator was installed on amine-terminated magnetic Dynabeads via the reaction of the amine groups with $\alpha$ bromoisobutyryl bromide, followed by ATRP for each of the monomers. ${ }^{29}$ XPS wide scan spectra and characteristic narrow scan spectra are shown in Figure 1 for each of the polymercoated beads (for quantitative evaluation of wide scans and additional narrow scan spectra, see Figures S14, S16, S18, S20, and S22).

XPS spectra show a similar composition for the polymercoated beads as compared to the coated gold surfaces (with the logical exception that no $\mathrm{Au} 4 \mathrm{f}$ peak is seen in the wide scans of beads). The poly(SBMAA-3)- and poly(SBMAA-2)-coated beads display two minor silicon peaks at 149 and $100 \mathrm{eV}$, which correspond to the underlying $\mathrm{Si}(111)$ surface on which the beads were deposited for the XPS measurement. The characteristic 1:1 ratio of the nitrogen peaks in the $\mathrm{N} 1 \mathrm{~s}$ spectrum, corresponding to the quaternary ammonium at 401-402 eV and the amide nitrogen at 398-399 eV, were clearly observed for poly(SBMAA-3) and poly(SBMAA-2)coated beads.

This 1:1 ratio was not obtained for poly(CBMAA-2)-coated beads; at best, $44 \%$ ammonium versus $56 \%$ for the amide was found. This is in contrast to the results obtained for the poly(CBMAA-2)-coated gold surfaces. It was not caused by an insufficient thickness of the polymer layer but turned out to be a time-dependent degradation of the poly(CBMAA-2) layers within the XPS. This was revealed by the XPS analysis of poly(CBMAA-2) brushes that were grown for $12 \mathrm{~min}$, as in the standard procedure, but in additional experiments also grown for 30 and $90 \mathrm{~min}$ on both gold surfaces and beads. However, in none of these was the 1:1 ammonium to amide peak ratio observed (see Figure S23). On the basis of the XPS spectra, we speculated that there was an effect of the duration of time between sample preparation and XPS analysis on the nitrogen ratio. For evaluating this unexpected observation, the $12 \mathrm{~min}$ gold sample was measured twice without having the sample taken out of the ultrahigh vacuum (UHV) chamber of the XPS, with $12 \mathrm{~h}$ in between the two measurements. In the second measurement, the ammonium peak had become appreciably smaller (see Figure 2), indicating that the poly(CBMAA-2)

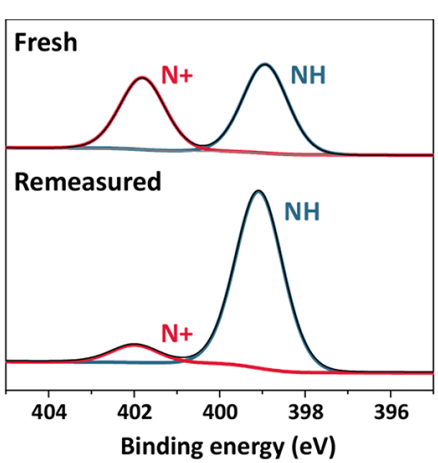

Figure 2. XPS N 1s narrow scan of a poly(CBMAA-2)-coated gold surface grown via $12 \mathrm{~min}$ of SI-ATRP, measured directly after preparation (fresh), and remeasured after $\sim 12 \mathrm{~h}$ (remeasured) within the same XPS measurement (i.e., without taking the sample out of the UHV chamber of the XPS apparatus).

coatings degrade over a relatively short period of time within the vacuum of the XPS. This also explains why an intact CBMAA-2 brush could be measured on a gold substrate but not on a bead substrate as the gold surfaces could be directly measured after preparation while the beads were first dropcast on a silicon substrate and dried for several hours in a vacuum oven. The disappearance of the ammonium peak was also observed for poly(CBMAA-2)-coated gold surfaces upon storage for several days in an argon glovebox (data not shown).

As the brush thicknesses stayed more or less the same (see Figure S24), and the relative nitrogen to carbon-oxygen percentages slightly increased upon storage, we hypothesize that an elimination reaction occurred in which a $\mathrm{C}_{2} \mathrm{H}_{3} \mathrm{COO}^{-}$ group is eliminated and a tertiary amine remains on the polymer brush (and thereby loses its quaternary character). It has been reported that CB-2 monomers and resulting polymers are unstable in both acid and base and can undergo a Hofmann elimination. $^{42-44}$ A similar degradation process was not observed for the sulfobetaine or phosphocholine polymer brushes; it is currently not known whether the degradation we observed is specific for the UHV conditions required for the XPS analysis or also takes place to a significant degree upon prolonged storage in other media. Whereas CB-2-based polymer layers have been established as one of the best antifouling coatings, ${ }^{2}$ this type of degradation has, to the best of our knowledge, never been evaluated in relation to the corresponding antifouling properties. The beads that were used for antifouling studies were kept in an aqueous solution at all 
steps and did not undergo any drying phase as is required for XPS analysis.

Antifouling Performance. We have previously shown that the antifouling performance and specific binding capabilities of polymer coatings can be reliably evaluated using a bead-based platform with flow cytometry as the readout system. ${ }^{29}$ Flow cytometry allows for the automated measurement of thousands of micrometer-sized particles per second, enabling quick analysis and good statistics. ${ }^{45}$ The obtained poly(SBMAA-3)-, poly(SBMAA-2)-, poly(CBMAA2)-, poly(PCMA-2)-, and poly(HPMAA)-coated beads were compared for their antifouling performance using this platform. To this end, the polymer-coated beads were either incubated in PBS, a solution containing fluorescently labeled BSA, or a biotinylated $10 \%$ serum solution. Beads incubated with biotinylated serum were subsequently stained with fluorescently labeled streptavidin (see Figure 3 for a schematic

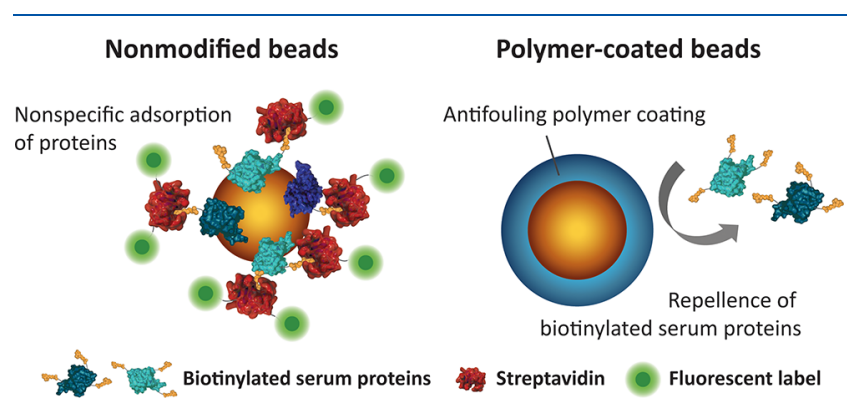

Figure 3. Schematic representation of antifouling experiments on nonmodified and polymer-coated beads using biotinylated serum proteins. Nonspecifically adsorbed serum proteins on the beads are subsequently incubated with fluorescently labeled streptavidin. The fluorescence intensity reflects the amount of adsorbed proteins. Polymer-coated beads largely repel the serum proteins and therefore show low fluorescence intensities.

representation). We chose to first biotinylate the serum proteins because a higher fluorescence signal, and therefore sensitivity, could be obtained as compared to the directly fluorescently labeled serum used in our previous study. ${ }^{29}$ This we rationalize by noting that the serum proteins can be equipped with multiple biotin units, which can all be bound by streptavidin, and each streptavidin can in turn have multiple fluorescent groups (see Figure 3), and by the higher fluorescence of PE compared to that of HLF-488. The increase in sensitivity enabled us to discriminate a good performing antifouling layer from an excellent one.

For each sample, the median fluorescence intensity (MFI) was corrected for the autofluorescence of the beads by subtracting the MFI value of a bead sample that was incubated in only PBS (see Tables S1 and S2 for uncorrected values). The corrected MFI values are plotted in Figure 4. The more fluorescently labelled proteins get adsorbed onto the beads, the higher the fluorescence intensity of those beads. Hence, a higher fluorescence intensity corresponds to a higher degree of fouling. All polymer-coated beads can clearly suppress the nonspecific adsorption of proteins as compared to the nonmodified (NM) beads (more than 2 orders of magnitude for BSA and 1 order of magnitude for $10 \%$ serum). It can be concluded that all polymer-coated beads were able to suppress the adsorption of BSA to levels within the experimental noise. Although in itself a useful result, antifouling properties toward single protein solutions do not allow extrapolation toward the antifouling behavior in more complex biological media like serum solutions. ${ }^{10}$ When the beads were incubated with the $10 \%$ serum solution, a fouling solution that can be considered as typical for the fouling obtained in, e.g., medical diagnostics, differences between the monomers could be observed. These observations lead to the following ranking of antifouling performance based on the amount of adsorbed serum proteins: HPMAA $\geq$ CBMAA- $\approx$ PCMA-2 $>$ SBMAA- 2 SBMAA-3 $\gg$ nonmodified beads.

Factors Determining the Antifouling Properties of Polymer Brushes. Considering the two sulfobetaine-based coatings, poly(SBMAA-2)-coated beads performed better (factor 2.8 ) in a $10 \%$ serum solution than poly(SBMAA-3)coated beads, which should be attributed to the difference in carbon spacer length between the charges. This is in line with studies that investigated the antifouling behavior of poly(carboxybetaines $)^{10,13}$ and poly $(N$-hydroxyl alkyl amide) materials $^{20,46}$ with varying carbon spacer length: a shorter distance between the charges results in better antifouling

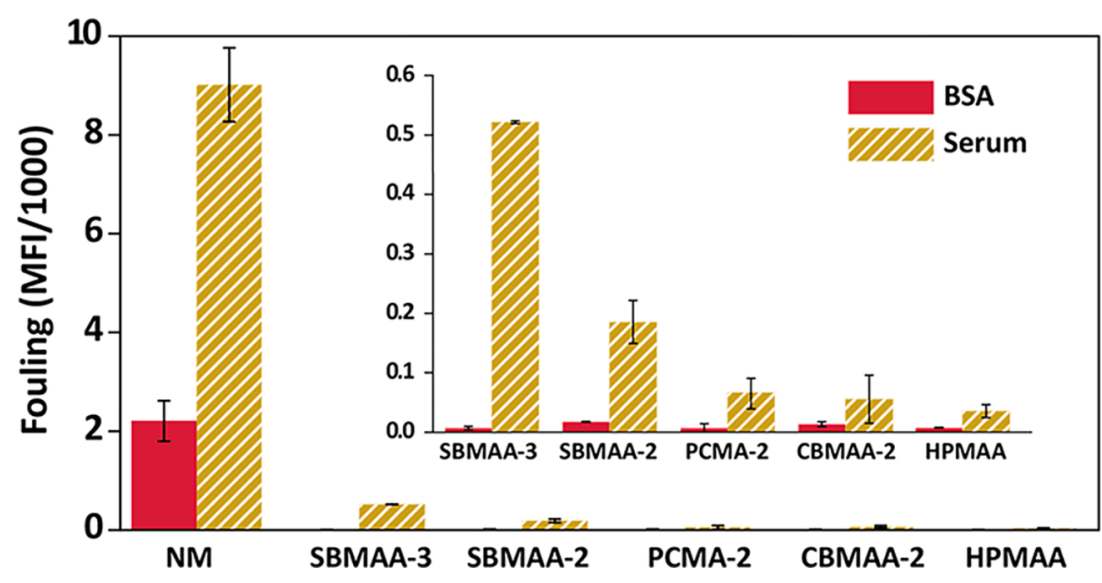

Figure 4. Antifouling performance of polymer-coated Dynabeads measured by flow cytometry. Nonmodified beads (NM) and polymer brushcoated beads were incubated with either (a) fluorescently labeled BSA $(0.5 \mathrm{mg} / \mathrm{mL})$ or (b) biotinylated serum $(10 \%$ serum solution, $\sim 6 \mathrm{mg} / \mathrm{mL})$ and fluorescently labeled streptavidin. The median fluorescence intensity (MFI) of the beads was corrected for the MFI values obtained for beads incubated in PBS. Presented data are averages from at least duplicates of independently performed experiments. Standard deviations are presented as error bars. The inset shows a zoom-in of the same data. 
properties. Conversely, it was found by Wang et al. ${ }^{25}$ that poly(SB-3), compared to poly(SB-2) and poly(SB-4), is best in preventing nonspecific BSA adsorption at nonphysiologically low ionic strengths, whereas at salt concentrations $>0.1 \mathrm{M}$, no differences were seen in BSA adsorption. It has been shown that the behavior of poly $(\mathrm{SB})$ materials is highly dependent on the ionic strength as the intra/interchain associations between sulfobetaine units can be disrupted by the addition of salt, leading to swelling of the polymer brush. ${ }^{24-26}$ The combination of our data and literature data leads to the following picture: with smaller CSL (fewer hydrophobic methylene moieties), the interaction between oppositely charged groups is stronger. This charge-charge interaction can be weakened by increasing the salt concentration. For larger CSL, only a small amount of salt is required, whereas for, e.g., SBMAA-2, higher ionic strengths will be required. Once swelling takes places, the hydration of such closely spaced charged [as in poly(SB-2) brushes] is also increased, and as a result, such brushes are more diffuse and swollen than poly(SB-3) brushes at $100 \mathrm{mM} \mathrm{NaCl}$ concentration. This suggests that, in the low ionic strength regime, poly (SB-3) brushes are more swollen/better hydrated than poly(SB-2) brushes and this is in line with the finding that their antifouling performance under these conditions is better than for poly(SB2) (as indeed found by Wang et al. ${ }^{25}$ ). Although relevant for understanding, this regime is of little practical importance as in most biologically relevant samples the ionic strength is $>0.1 \mathrm{M}$. In contrast, poly(SB-2) brushes become more swollen than poly(SB-3) brushes in the $>0.1 \mathrm{M}$ salt regime and should therefore perform better at higher salt concentrations (Figure 4). To study the differences between poly(SB-2) and poly(SB3 ) in more detail, the difference between SBMAA-3 and SBMAA-2 was also evaluated using surface plasmon resonance (SPR), a commonly used method to study antifouling. Figure 5

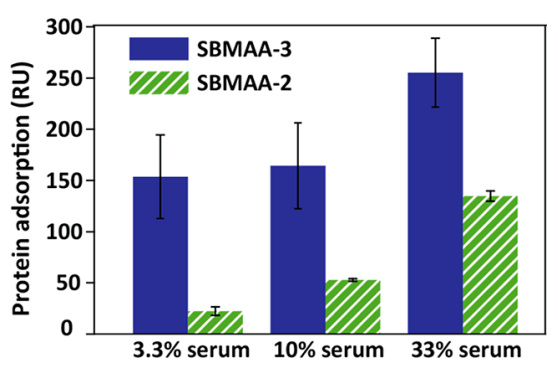

Figure 5. Protein adsorption from $3.3,10$, or $33 \%$ cow serum on poly(SBMAA-3)- and poly(SBMAA-2)-coated gold surfaces as measured by SPR. Averages of three independent measurements are shown; error bars represent standard deviations of those measurements. The SPR data were obtained using dialyzed serum solutions as otherwise artifacts were seen in the sensorgrams caused by differences in salt concentration (see Supporting Information for further explanation).

confirms that, when using $3.3,10$, or $33 \%$ serum solutions, SBMAA-2 consistently outperforms SBMAA-3. In addition, the relative difference is bigger for more dilute samples, and like in the bead assay at $10 \%$ serum, SBMAA- 2 adsorbs $\sim 3 \times$ less protein than SBMAA-3 (see also Figure 4). This also shows the relative quantitative agreement between these techniques.

Besides the comparison between SBMAA-2 and SBMAA-3, Figure 4 also reveals the differences between SBMAA- 2 and CBMAA-2; this data constitute, to the best of our knowledge, the first direct experimental comparison of the antifouling behavior of polymer brushes derived from SB-2 or CB-2 monomers. Figure 4 shows that poly(CBMAA-2) performs better than poly(SBMAA-2) in a $10 \%$ serum solution. As the chemical structures of CBMAA-2 and SBMAA-2 are the same except for their anionic group, it can be concluded that as anionic group carboxylates outperform sulfonates. Sulfonate anions are larger than carboxylates and have their negative charge distributed over more oxygen atoms. As a result, it is expected that more water will surround sulfonates than carboxylates, but that water will be more strongly bound by carboxylates. This qualitative picture has indeed been simulated accurately by Jiang and co-workers. ${ }^{47}$ Together, this suggests that a material with few but tightly bound water molecules is more effective in resisting nonspecific adsorption of proteins than a material with more water molecules that are loosely bound. For the properties of the polymer brushes to be assessed further, the zeta potential of the polymer-coated beads was also measured (see Figure S25). All polymer-coated beads yielded moderately negative zeta potentials, as was previously reported for zwitterionic particles. ${ }^{48-50}$ The zeta potentials for poly(SBMAA-3), poly(SBMAA-2), and poly(CBMAA-2) are within the same range: $-9.7 \pm 0.7,-8.2 \pm 1.1$, and $-11.1 \pm$ 0.6 , respectively. Given the similarity in their zeta potentials, the observed difference in antifouling capacity of the beads cannot be accounted for by differences in the surface charge of the sulfobetaine- and carboxybetaine-coated beads. It is unclear whether the aforementioned degradation of the poly(CBMAA2) layers in vacuum or inert atmosphere plays a role in the obtained zeta potential and antifouling properties of this material. As seen in Figure 4, poly(HPMAA) performs better than the sulfobetaines and slightly better than poly(CBMAA2) and poly(PCMA-2). That poly(HPMAA) performs similar or better than poly(CBMAA-2) is consistent with the literature. ${ }^{19,27}$ Why HPMAA and other simple hydroxyl methacrylamide monomers perform so well is not entirely understood. In comparison to zwitterionic brushes, they are only moderately hydrophilic, and ionic solvation, as occurs for zwitterionic materials, is stronger than hydrogen-bonding solvation. ${ }^{17,19}$ It might be related to tightly bound water molecules by hydrogen bond bridges that can be formed between one water molecule and the hydroxyl and amide group of the same monomer unit. Preliminary quantum chemical calculations on model systems $(B 97 / 6-311+G(d, p)$ using a PCM self-consistent reaction field model to simulate water) yield complexation energies of a HPMAA unit and water, bound by two hydrogen bonds (Figure 6), of 6-12 $\mathrm{kcal} / \mathrm{mol}$. These data indeed point to strong hydration of HMPAA and, in combination with the antifouling data, indicate the usefulness of deeper analyses. Besides the hydration properties, the low surface charge of poly(HPMAA)-coated beads may also contribute to its excellent antifouling properties; poly(HPMAA)-coated beads showed the lowest zeta potential of the five monomers tested within this study (see Figure S25).

Similar to the hydroxyl acrylamide monomers, phosphocholines are not often compared to other types of antifouling polymer brushes. In a study by Rodriguez-Emmenegger, ${ }^{27}$ it was found that poly(PC) performed less well than poly (SB-3) and poly(CB-2); in contrast, we observe that poly(PCMA-2) performed better than poly(SBMAA-3) and equally good as poly(CBMAA-2). Noteworthily, the zeta potential of poly(PCMA-2) is lower than for the sulfobetaine- and carboxybetaine-coated beads (see Figure S25). 


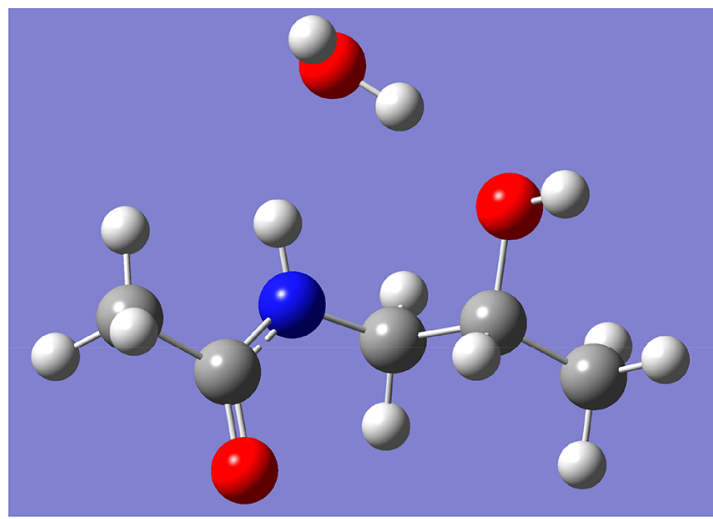

Figure 6. Hydration of an HPMAA model via H-bonds consisting of $\mathrm{N}-\mathrm{H} \cdots \mathrm{O}$ and $\mathrm{O}-\mathrm{H} \cdots \mathrm{H}$ interactions (B97D/6-311+G(d,p)-optimized structure; see text for details).

On the basis of our findings, we thus conclude that poly(HPMAA)-coated beads are best capable of resisting nonspecific protein adsorption from BSA and serum solutions, whereas all zwitterionic brushes are good but not as good. In selecting the optimal antifouling coating, besides the actual antifouling performance, several other factors come into play depending on the application. (1) $p H$ of the medium: the antifouling performance of poly(CBMAA-2) is $\mathrm{pH}$-dependent ${ }^{14}$ as the zwitterionic character is lost upon protonation of the carboxylic acid at low $\mathrm{pH}$. (2) Ionic strength and temperature: the antifouling characteristics of sulfobetaines are highly dependent on ionic strength and temperature $e^{23-25,51}$ due to strong intra/interchain interactions between the sulfobetaine moieties. These are so strong that, without any added ions, sulfobetaine-based polymers can even be used as the basis of self-healing antifouling materials. ${ }^{52,53}$ (3) Biofunctionalization: CBMAA-2 and HPMAA are the only monomers that can directly be functionalized within the brush with biorecognition elements, ${ }^{2,19}$ albeit at the cost of diminished antifouling properties; ${ }^{54}$ for sulfobetaines, an efficient synthesis of azide-functionalized monomers is available that allows azide-alkyne click-based biofunctionalizations to take place without loss of the zwitterionic character. ${ }^{33}$ (4) Ease of use: The growth of sulfobetaine brushes is extremely reproducible and allows for minor deviations from the protocol. Growing HPMAA brushes, on the other hand, turned out to be more challenging and was found to be more sensitive to oxygen contamination. Further optimization of the synthetic protocols would be well deserved given the antifouling characteristics. PCMA-2 easily self-polymerizes ${ }^{55}$ and was therefore slightly less robust in use than the sulfobetaines. (5) FDA approval: Of the materials under study, only PCMA-2-based brushes are currently FDA approved for biomedical applications. ${ }^{16}$ (6) Characterization: Poly(CBMAA-2) brush characterization was challenging due to the aforementioned degradation process during UHV analysis. These six factors involved in the use of these different antifouling brushes imply that further research into this field is both required and worthwhile.

\section{CONCLUSIONS}

In this study, we systematically compared five hydrogenbonding and zwitterionic polymer brushes for their antifouling performance using a bead-based assay. In essence, all brushes fully prevent the nonspecific adsorption in single-protein BSA solutions. In solutions containing more complex protein mixtures (e.g., a $10 \%$ serum solution), fouling is reduced by at least an order of magnitude as compared to nonmodified beads, but no complete antifouling is observed for any monomer. Our observations lead to the following antifouling ranking based on the amount of adsorbed serum proteins: HPMAA $\geq$ CBMAA-2 $\approx$ PCMA-2 $>$ SBMAA-2 > SBMAA-3 $\gg$ nonmodified beads.

Each brush has its own advantages and disadvantages, which may direct the preferred use in different situations. Of the family of sulfobetaines, we show for the first time that poly(SBMAA-2) performs consistently better in antifouling studies than poly(SBMAA-3) due to the reduced spacer length between opposing charges. The excellent performance of poly(HPMAA), equal to or better than any of the zwitterionic monomers under study, is likely related to strong and multiple hydrogen bond formation and/or low surface charge.

We have shown that our bead-based platform is suitable for screening different antifouling coatings for their antifouling capabilities. As thousands of beads can be prepared and analyzed at once, it is a valuable and statistically robust method to measure the antifouling performance of polymer-coated beads in detail.

\section{ASSOCIATED CONTENT}

\section{S Supporting Information}

The Supporting Information is available free of charge on the ACS Publications website at DOI: 10.1021/acs.langmuir.8b01832.

Monomer characterization, additional XPS spectra, zeta potential measurements, additional flow cytometry data, and SPR experiments (PDF)

\section{AUTHOR INFORMATION}

\section{Corresponding Author}

*E-mail: Han.Zuilhof@wur.nl.

ORCID $\odot$

Esther van Andel: 0000-0001-8404-688X

Sidharam P. Pujari: 0000-0003-0479-8884

Maarten M. J. Smulders: 0000-0002-6855-0426

Han Zuilhof: 0000-0001-5773-8506

Notes

The authors declare no competing financial interest.

\section{ACKNOWLEDGMENTS}

The authors thank Digvijay Gahtory, Judith Firet, and Luc Scheres for their help regarding the synthesis of the SBMAA-2 and CBMAA-2 monomers, Andriy Kuzmyn for assistance with the polymerization of HPMAA, René Achterberg for his help using the Biacore SPR apparatus, Remco Fokkink for his input on the zeta potential measurements, and Ben Meijer for his assistance with the flow cytometer. This work was supported by NanoNextNL (program 3E), a micro- and nanotechnology consortium of the government of The Netherlands and 130 partners.

\section{REFERENCES}

(1) Horbett, T. A. The Role of Adsorbed Proteins in Animal-Cell Adhesion. Colloids Surf., B 1994, 2, 225-240.

(2) Jiang, S. Y.; Cao, Z. Q. Ultralow-Fouling, Functionalizable, and Hydrolyzable Zwitterionic Materials and Their Derivatives for Biological Applications. Adv. Mater. 2010, 22, 920-932. 
(3) Chae, K. H.; Jang, Y. M.; Kim, Y. H.; Sohn, O. J.; Rhee, J. I. AntiFouling Epoxy Coatings for Optical Biosensor Application Based on Phosphorylcholine. Sens. Actuators, B 2007, 124, 153-160.

(4) Donlan, R. M. Biofilms and Device-Associated Infections. Emerging Infect. Dis. 2001, 7, 277-281.

(5) Chapman, R. G.; Ostuni, E.; Takayama, S.; Holmlin, R. E.; Yan, L.; Whitesides, G. M. Surveying for Surfaces That Resist the Adsorption of Proteins. J. Am. Chem. Soc. 2000, 122, 8303-8304.

(6) Ostuni, E.; Chapman, R. G.; Holmlin, R. E.; Takayama, S.; Whitesides, G. M. A Survey of Structure-Property Relationships of Surfaces That Resist the Adsorption of Protein. Langmuir 2001, 17, $5605-5620$.

(7) Wei, Q.; Becherer, T.; Angioletti-Uberti, S.; Dzubiella, J.; Wischke, C.; Neffe, A. T.; Lendlein, A.; Ballauff, M.; Haag, R. Protein Interactions with Polymer Coatings and Biomaterials. Angew. Chem., Int. Ed. 2014, 53, 8004-8031.

(8) Prime, K. L.; Whitesides, G. M. Self-Assembled Organic Monolayers - Model Systems for Studying Adsorption of Proteins at Surfaces. Science 1991, 252, 1164-1167.

(9) Li, L. Y.; Chen, S. F.; Zheng, J.; Ratner, B. D.; Jiang, S. Y. Protein Adsorption on Oligo(Ethylene Glycol)-Terminated Alkanethiolate Self-Assembled Monolayers: The Molecular Basis for Nonfouling Behavior. J. Phys. Chem. B 2005, 109, 2934-2941.

(10) Ladd, J.; Zhang, Z.; Chen, S.; Hower, J. C.; Jiang, S. Zwitterionic Polymers Exhibiting High Resistance to Nonspecific Protein Adsorption from Human Serum and Plasma. Biomacromolecules 2008, 9, 1357-1361.

(11) Nguyen, A. T.; Baggerman, J.; Paulusse, J. M. J.; van Rijn, C. J. M.; Zuilhof, H. Stable Protein-Repellent Zwitterionic Polymer Brushes Grafted from Silicon Nitride. Langmuir 2011, 27, 25872594.

(12) Kim, J. C.; Kim, M.; Jung, J.; Kim, H.; Kim, I. J.; Kim, J. R.; Ree, M. Biocompatible Characteristics of Sulfobetaine-Containing Brush Polymers. Macromol. Res. 2012, 20, 746-753.

(13) Vaisocherova, H.; Zhang, Z.; Yang, W.; Cao, Z.; Cheng, G.; Taylor, A. D.; Piliarik, M.; Homola, J.; Jiang, S. Functionalizable Surface Platform with Reduced Nonspecific Protein Adsorption from Full Blood Plasma-Material Selection and Protein Immobilization Optimization. Biosens. Bioelectron. 2009, 24, 1924-30.

(14) Zhang, Z.; Vaisocherova, H.; Cheng, G.; Yang, W.; Xue, H.; Jiang, S. Y. Nonfouling Behavior of Polycarboxybetaine-Grafted Surfaces: Structural and Environmental Effects. Biomacromolecules 2008, 9, 2686-2692.

(15) Ning, J. Y.; Li, G.; Haraguchi, K. Synthesis of Highly Stretchable, Mechanically Tough, Zwitterionic Sulfobetaine Nanocomposite Gels with Controlled Thermosensitivities. Macromolecules 2013, 46, 5317-5328.

(16) Iwasaki, Y.; Ishihara, K. Cell Membrane-Inspired Phospholipid Polymers for Developing Medical Devices with Excellent Biointerfaces. Sci. Technol. Adv. Mater. 2012, 13, 064101.

(17) Chen, H.; Zhao, C.; Zhang, M. Z.; Chen, Q.; Ma, J.; Zheng, J. Molecular Understanding and Structural-Based Design of Polyacrylamides and Polyacrylates as Antifouling Materials. Langmuir 2016, 32, 3315-3330.

(18) Pereira, A. D.; Rodriguez-Emmenegger, C.; Surman, F.; Riedel, T.; Alles, A. B.; Brynda, E. Use of Pooled Blood Plasmas in the Assessment of Fouling Resistance. RSC Adv. 2014, 4, 2318-2321.

(19) Rodriguez-Emmenegger, C.; Brynda, E.; Riedel, T.; Houska, M.; Subr, V.; Alles, A. B.; Hasan, E.; Gautrot, J. E.; Huck, W. T. S. Polymer Brushes Showing Non-Fouling in Blood Plasma Challenge the Currently Accepted Design of Protein Resistant Surfaces. Macromol. Rapid Commun. 2011, 32, 952-957.

(20) Yang, J.; Zhang, M.; Chen, H.; Chang, Y.; Chen, Z.; Zheng, J. Probing the Structural Dependence of Carbon Space Lengths of Poly(N-Hydroxyalkyl Acrylamide)-Based Brushes on Antifouling Performance. Biomacromolecules 2014, 15, 2982-91.

(21) Vaisocherova, H.; Zhang, Z.; Yang, W.; Cao, Z. Q.; Cheng, G.; Taylor, A. D.; Piliarik, M.; Homola, J.; Jiang, S. Y. Functionalizable Surface Platform with Reduced Nonspecific Protein Adsorption from
Full Blood Plasma-Material Selection and Protein Immobilization Optimization. Biosens. Bioelectron. 2009, 24, 1924-1930.

(22) Shao, Q.; Jiang, S. Y. Effect of Carbon Spacer Length on Zwitterionic Carboxybetaines. J. Phys. Chem. B 2013, 117, 13571366.

(23) Shao, Q.; Jiang, S. Y. Molecular Understanding and Design of Zwitterionic Materials. Adv. Mater. 2015, 27, 15-26.

(24) Higaki, Y.; Inutsuka, Y.; Sakamaki, T.; Terayama, Y.; Takenaka, A.; Higaki, K.; Yamada, N. L.; Moriwaki, T.; Ikemoto, Y.; Takahara, A. Effect of Charged Group Spacer Length on Hydration State in Zwitterionic Poly(Sulfobetaine) Brushes. Langmuir 2017, 33, 84048412.

(25) Wang, T.; Kou, R.; Liu, H.; Liu, L.; Zhang, G.; Liu, G. Anion Specificity of Polyzwitterionic Brushes with Different Carbon Spacer Lengths and Its Application for Controlling Protein Adsorption. Langmuir 2016, 32, 2698-2707.

(26) Shao, Q.; Mi, L.; Han, X.; Bai, T.; Liu, S.; Li, Y.; Jiang, S. Differences in Cationic and Anionic Charge Densities Dictate Zwitterionic Associations and Stimuli Responses. J. Phys. Chem. B 2014, 118, 6956-62.

(27) Rodriguez Emmenegger, C.; Brynda, E.; Riedel, T.; Sedlakova, Z.; Houska, M.; Alles, A. B. Interaction of Blood Plasma with Antifouling Surfaces. Langmuir 2009, 25, 6328-6333.

(28) Ueda, T.; Oshida, H.; Kurita, K.; Ishihara, K.; Nakabayashi, N. Preparation of 2-Methacryloyloxyethyl Phosphorylcholine Copolymers with Alkyl Methacrylates and Their Blood Compatibility. Polym. J. 1992, 24, 1259-1269.

(29) van Andel, E.; de Bus, I.; Tijhaar, E. J.; Smulders, M. M. J.; Savelkoul, H. F. J.; Zuilhof, H. Highly Specific Binding on Antifouling Zwitterionic Polymer-Coated Microbeads as Measured by Flow Cytometry. ACS Appl. Mater. Interfaces 2017, 9, 38211-38221.

(30) Chen, L.; Honma, Y.; Mizutani, T.; Liaw, D. J.; Gong, J. P.; Osada, Y. Effects of Polyelectrolyte Complexation on the UCST of Zwitterionic Polymer. Polymer 2000, 41, 141-147.

(31) Rodriguez-Emmenegger, C.; Schmidt, B. V. K. J.; Sedlakova, Z.; Subr, V.; Alles, A. B.; Brynda, E.; Barner-Kowollik, C. Low Temperature Aqueous Living/Controlled (Raft) Polymerization of Carboxybetaine Methacrylamide up to High Molecular Weights. Macromol. Rapid Commun. 2011, 32, 958-965.

(32) Joshi, S.; Pellacani, P.; van Beek, T. A.; Zuilhof, H.; Nielen, M. W. F. Surface Characterization and Antifouling Properties of Nanostructured Gold Chips for Imaging Surface Plasmon Resonance Biosensing. Sens. Actuators, B 2015, 209, 505-514.

(33) Lange, S. C.; van Andel, E.; Smulders, M. M. J.; Zuilhof, H. Efficient and Tunable Three-Dimensional Functionalization of Fully Zwitterionic Antifouling Surface Coatings. Langmuir 2016, 32, 10199-10205.

(34) Hofmann, S. Quantitative Analysis (Data Evaluation). In Augerand X-Ray Photoelectron Spectroscopy in Materials Science; SpringerVerlag: Berlin/Heidelberg, 2013; p 137.

(35) Petrovykh, D. Y.; Kimura-Suda, H.; Tarlov, M. J.; Whitman, L. J. Quantitative Characterization of DNA Films by X-Ray Photoelectron Spectroscopy. Langmuir 2004, 20, 429-440.

(36) Gauthier, M.; Carrozzella, T.; Penlidis, A. Sulfobetaine Zwitterionomers Based Onn-Butyl Acrylate and 2-Ethoxyethyl Acrylate: Monomer Synthesis and Copolymerization Behavior. J. Polym. Sci., Part A: Polym. Chem. 2002, 40, 511-523.

(37) Ning, J.; Li, G.; Haraguchi, K. Synthesis of Highly Stretchable, Mechanically Tough, Zwitterionic Sulfobetaine Nanocomposite Gels with Controlled Thermosensitivities. Macromolecules 2013, 46, 53175328.

(38) Tang, Y.; Lu, J. R.; Lewis, A. L.; Vick, T. A.; Stratford, P. W. Swelling of Zwitterionic Polymer Films Characterized by Spectroscopic Ellipsometry. Macromolecules 2001, 34, 8768-8776.

(39) Blaszykowski, C.; Sheikh, S.; Thompson, M. A Survey of Stateof-the-Art Surface Chemistries to Minimize Fouling from Human and Animal Biofluids. Biomater. Sci. 2015, 3, 1335-1370. 
(40) Matyjaszewski, K. Atom Transfer Radical Polymerization (Atrp): Current Status and Future Perspectives. Macromolecules 2012, 45, 4015-4039.

(41) Yildirim, E.; Cimen, D.; Zengin, A.; Caykara, T. Synthesis of Poly(N-(2-Hydroxypropyl) Methacrylamide) Brushes by InterfaceMediated Raft Polymerization. RSC Adv. 2016, 6, 45259-45264.

(42) Chevalier, Y.; Storet, Y.; Pourchet, S.; Leperchec, P. Tensioactive Properties of Zwitterionic Carboxybetaine Amphiphiles. Langmuir 1991, 7, 848-853.

(43) Wielema, T. A.; Engberts, J. B. F. N. Zwitterionic Polymers 0.2. Synthesis of a Novel Series of Poly(Vinylbetaines) and the Effect of the Polymeric Structure on the Solubility Behavior in Water. Eur. Polym. J. 1990, 26, 415-421.

(44) Cao, B.; Li, L. L.; Tang, Q.; Cheng, G. The Impact of Structure on Elasticity, Switchability, Stability and Functionality of an All-inOne Carboxybetaine Elastomer. Biomaterials 2013, 34, 7592-7600.

(45) Shapiro, H. M. Practical Flow Cytometry, 4th ed.; WILEY-LISS: Hoboken, NJ, 2003.

(46) Chen, S. F.; Li, L. Y.; Zhao, C.; Zheng, J. Surface Hydration: Principles and Applications toward Low-Fouling/Nonfouling Biomaterials. Polymer 2010, 51, 5283-5293.

(47) Shao, Q.; He, Y.; White, A. D.; Jiang, S. Y. Difference in Hydration between Carboxybetaine and Sulfobetaine. J. Phys. Chem. B 2010, 114, 16625-16631.

(48) Jiang, W.; Fischer, G.; Girmay, Y.; Irgum, K. Zwitterionic Stationary Phase with Covalently Bonded Phosphorylcholine Type Polymer Grafts and Its Applicability to Separation of Peptides in the Hydrophilic Interaction Liquid Chromatography Mode. J. Chromatogr., A 2006, 1127, 82-91.

(49) Guo, S.; Jańczewski, D.; Zhu, X.; Quintana, R.; He, T.; Neoh, K. G. Surface Charge Control for Zwitterionic Polymer Brushes: Tailoring Surface Properties to Antifouling Applications. J. Colloid Interface Sci. 2015, 452, 43-53.

(50) Weng, X.-D.; Bao, X.-J.; Jiang, H.-D.; Chen, L.; Ji, Y.-L.; An, Q.F.; Gao, C.-J. Ph-Responsive Nanofiltration Membranes Containing Carboxybetaine with Tunable Ion Selectivity for Charge-Based Separations. J. Membr. Sci. 2016, 520, 294-302.

(51) Gauthier, M.; Carrozzella, T.; Snell, G. Sulfobetaine Zwitterionomers Based on N-Butyl Acrylate and 2-Ethoxyethyl Acrylate: Physical Properties. J. Polym. Sci., Part B: Polym. Phys. 2002, 40, 2303-2312.

(52) Wang, Z.; van Andel, E.; Pujari, S. P.; Feng, H.; Dijksman, J. A.; Smulders, M. M. J.; Zuilhof, H. Water-Repairable Zwitterionic Polymer Coatings for Anti-Biofouling Surfaces. J. Mater. Chem. B 2017, 5, 6728-6733.

(53) Wang, Z.; Fei, G.; Xia, H.; Zuilhof, H. Dual Water-Healable Zwitterionic Polymer Coatings for Anti-Biofouling Surfaces. J. Mater. Chem. B 2018, DOI: $10.1039 / \mathrm{c} 8 \mathrm{tb} 01863 \mathrm{~d}$, in press

(54) Lisalova, H.; Brynda, E.; Houska, M.; Visova, I.; Mrkvova, K.; Song, X. C.; Gedeonova, E.; Surman, F.; Riedel, T.; Pop-Georgievski, O.; Homola, J. Ultralow-Fouling Behavior of Biorecognition Coatings Based on Carboxy-Functional Brushes of Zwitterionic Homo- and Copolymers in Blood Plasma: Functionalization Matters. Anal. Chem. 2017, 89, 3524-3531.

(55) Lobb, E. J.; Ma, I.; Billingham, N. C.; Armes, S. P.; Lewis, A. L. Facile Synthesis of Well-Defined, Biocompatible PhosphorylcholineBased Methacrylate Copolymers Via Atom Transfer Radical Polymerization at 20 Degrees C. J. Am. Chem. Soc. 2001, 123, 7913-7914. 